\title{
The Paradoxical Nature of Electronic Decision Aids on Comparison-Shopping: The Experiments and Analysis
}

\author{
Yun Wan ${ }^{1}$, Satya Menon ${ }^{2}$ and Arkalgud Ramaprasad ${ }^{3}$ \\ ${ }^{1}$ University of Houston, Victoria, wany@uhv.edu \\ ${ }^{2}$ Millward Brown, Inc., satya.menon@us.millwardbrown.com \\ ${ }^{3}$ University of Illinois at Chicago, prasad@uic.edu \\ Received 12 December 2008; received in revised form 5 May 2009; accepted 1 August 2009
}

\begin{abstract}
Consumers who use electronic decision aids such as comparison-shopping agents may be overwhelmed by the amount of choice information available to them, leading to an inability to choose or dissatisfaction with the ultimate choice, a state of "choice overload." Two experiments were designed to test the choice overload hypothesis. Eight choice tasks of different sizes were presented to subjects. By observing the decision quality, decision time, and decision confidence, we confirmed our hypothesis that choice overload exists when the comparison matrix exceeds a certain size (24 choices and 10 attributes for each choice in this research). Subjects were then given different combinations of decision-making tools (sorting and short-listing) and conditions (refreshing of information) to deal with the same choice task within the choice overload range obtained from the initial experiment. The use of both decision-making tools unexpectedly required more decision-making effort and resulted in less decision satisfaction than when only one decision-making tool was provided. We believe these findings are relevant to the future development of electronic decision aids. Further research is needed in this direction to extend our understanding of decision-making in electronic decision aids mediated environment.
\end{abstract}

Key words: Electronic Decision Aids, Choice Overload, Comparison-Shopping, Decision Support systems, Consumer Behavior 


\section{Introduction}

In the past five years, with the advent and popularity of Internet shopping, consumers have been increasingly faced with a variety of choices from online vendors. While traditional retail stores such as Wal-Mart may stock 100,000 items per location, a Web retailer such as Amazon.com may offer as many as 18 million items available to the consumer to select from. Meanwhile, the increasing availability and affordability of ecommerce technologies has encouraged more vendors' online presence. As a result, consumers are experiencing an unprecedented variety of choices offered by numerous vendors from the Web. So naturally, consumers are gradually coming to depend on the newly emerged electronic decision aids such as comparison-shopping agents for assistance, as explained by Clark [5] - [6].

Generally speaking, comparison-shopping agents, like other emerging electronic decision aids, are supposed to assist the consumer in choosing a product in a timely manner when there are many types, brands, and variations available in an online retail transaction. These agents are software applications that can interact with consumers and provide information aggregation and processing services upon request [7], [33]. They are expected to reduce the search cost of consumers and mitigate choice overload incurred by the increasing number of vendors as well as the products by narrowing the search for a product from multiple vendors, by assisting shoppers in comparing attributes, and by finding the best price or nearest point of distribution [14]. According to most recent survey by Nielsen, in January 2009, comparison-shopping engine NextTag.com ranked in the top 10 in all search engine traffic, including those general search engines like Google.

However, to maximize their profit and to accommodate the general public's insatiable demand for more choice information, comparison-shopping service providers usually encourage as many online vendors to join their comparison-shopping platform as possible. They also encourage online vendors to list as much product and product attribute information as possible. As a result, using these agents may increase the burden on the consumer who is given an overwhelming array of options, diminishing his or her ability to use the decision-making tools provided by the agent to process them, and subsequently undermining the intended benefit these agents are assumed to provide. This has a negative impact on both comparison-shopping service providers and online shoppers. So it is important to understand the paradoxical difference between "sufficient" choice information and "choice overload." The former is beneficial to both online shoppers and participating online vendors while the latter is a disservice to both of them, which may eventually lead to the decline of comparison-shopping services.

It is this paradoxical nature of electronic decision aids that we examined in this research. We demonstrate the existence of choice overload and frequent information updates by electronic decision aids may reduce the decision quality. We also demonstrate the inadequacy of decision tools like sorting and short-listing in addressing the choice overload problems. These findings provide important insights for the future development of comparison-shopping services.

The remainder of this paper is arranged as follows. First, we review previous research on the efficacy of electronic decision aids in individual decision-making and choice overload. Then we describe our research hypothesis and experiments' design. In our first experiment, we identify the existence of choice overload in the simulated online environment mediated by comparison-shopping agents. In the second experiment, we demonstrate the inadequacy of the two most frequently provided decision tools, short-listing and sorting, in counter-balancing the choice overload. Finally we discuss the implications of our experiments and suggestions for future research.

\section{Literature Review}

Consumer electronic decision aids are those electronic or computer-enabled information systems that could support the decision-making of consumers in an online environment by providing external decision information and decision tools [3], [34]. Research on the efficacy of consumer electronic decision aids has drawn increasing attention due to the B2C market development and the popularity of search engine marketing [8], [15] - [16], [26].

\subsection{Electronic Decision Aids, Comparison-Shopping Agents, and Decision Strategies}

Ariely [2] found that giving the consumers more control on information flow during the interaction with electronic decision aid would help them "better match their preferences, have better memory and knowledge about the domain they are examining, and be more confident in their judgments." But the study also found that controlling information flow also increases the demand of attention from consumers, thus may "have detrimental effects on consumers' ability to utilize information."

Electronic decision aids in online shopping could be roughly divided into two categories: the recommendation agent (RA) and the comparison matrix (CM) [1]. They were being used in two stages of decision-making, a recommendation agent (RA) in the first stage and a comparison matrix (CM) in the second stage. The RA allows the 
decision-maker to assign weights for attributes, and it then generates a product list for decision-making. The CM allows for side-by-side comparisons of products in terms of their attributes for information provided by the RA in the first stage. Häubl \& Trifts [9] found that RA and CM have strong favorable effects on both the quality and the efficiency of purchase decisions.

Olson \& Widing [16] found that even without specific weight assignment for attributes, decision aids that provide comparison information with equally weighted format performed as well or better in terms of overall decision quality. According to our observation, in an empirical online shopping environment, most electronic decision aids act as CM and don't solicit attribute weights information from online shoppers. So our focus of this study is mainly on CM or comparison-shopping agents.

Comparison-shopping agents, essentially more sophisticated CMs, are considered one major category of electronic decision aids being adopted by online shoppers. Typically, a comparison-shopping agent performs the functions of collecting product information based on the query of the shoppers, returning the product comparison matrix, and then providing shoppers with various decision strategies like elimination by aspect to identify the preferred choice [18]. Such strategies are performed with the appropriate decision tools that come with the decision task provided by the agents.

Theoretically, comparison-shopping agents can assist online shoppers to make quality shopping decisions by expanding their bounded rationality [22]. Online shoppers can reduce or eliminate searching costs as well as increase the information processing capability if they use these decision aids. Comparison-shopping agents that are conceptually well-designed seem to be able to achieve this optimal status [15]. In reality, however, comparisonshopping service providers have the motivation of listing more online vendors and their products, thus causing inflation of the choice information set, or comparison matrix. According to Payne, et al [17] and Todd, et al [27], people generally follow the least-effort principle [36] and tend to use non-compensatory heuristics when dealing with increasing information loads [11], [27] - [29].

These findings bring the efficacy provided by electronic decision aids under further scrutiny. This is because actualizing the potential benefit offered by electronic decision aids, such as comparison-shopping agents, depends on two conditions: 1) these decision aids are used adequately, and 2) the marginal cost of using these tools is less than the marginal benefit consumers gain from such usage. Only when both conditions are met would consumers use electronic decision aids. Due to least-effort propensity by consumers, researchers found that when individuals use decision aids, they tend to switch from more effortful compensatory strategies to less effortful non-compensative strategies when the decision task become more complex and/or the size of choice information that needs to be processed increases [17]. This means when comparison-shopping service providers inflated the CM by providing too much choice information, online shoppers may be overloaded by the choice information in the comparison matrix and use heuristics to avoid incurring more effort to process the decision task. The larger the size of inflated CM, the more impact from least effort principle and the more non-compensatory heuristics online shoppers tend to use. This may eventually lead to choice overload.

In the next section, we describe the symptoms of choice overload and discuss in detail how it can happen and how to observe it.

\subsection{Choice Overload}

Choice overload is a mental construct used to describe the feeling of being overwhelmed by an individual when he or she is confronted with too many choices [10], [20]. Based on Herbert Simon's bounded rationality theory [23, 24], we describe choice overload as a mental state in which the amount of choice information that needs to be processed exceeds the committed cognitive capacity of the decision-maker. As a result, the decision-maker cannot make a decision rationally.

Choice overload can be categorized as a sub-domain of information overload and the latter covers all kind of over provision of information while the former mainly refers to a set of formalized choices and each choice is characterized by a number of distinctive attributes.

It is difficult to measure choice overload directly, but many symptoms related to choice overload are observable. These include deferring the decision to buy [10], using too many heuristics when making the choice [12], or making poor decisions even when the decision tools provided by an agent enable the decision-maker to make an optimal choice. All these symptoms have been observed in traditional shopping environments and, in this research, are used as indicators of choice overload in the online environment. The last symptom is being used as one indicator of choice overload in this experiment.

Choice overload has been observed in the traditional shopping environment. In research by lyengar and Lepper [10] on choice overload, customers in a local grocery store were presented with two assortments of exotic jams to try. One had six different brands, and the other had 24 different brands. Customers were given a coupon so they could 
purchase the jelly they preferred later. It was found that though the extensive-choice condition induced more customers to stop (60\% vs. $40 \%$ ), the limited-choice condition actually resulted in more purchases (30\% vs. $3 \%$ ). The researchers concluded that it is the choice overload in the extensive-choice condition that made shoppers hesitates to buy because there were too many brands of jams to look over, and they were afraid of the regret they might feel if the forsaken options turned out to be better than the one actually chosen. In another recent study [21], individuals who tried to maximize utility in decisions by considering more options felt worse off and less satisfied with their decisions than individuals who made choices based on fewer alternatives.

Observations of counterintuitive behavior in online shopping environments in which shoppers were using various comparison-shopping agents could also be related to choice overload. For example, Brynjolfsson and Smith [4] found that when online shoppers used a comparison-shopping agent to search for price information on books and CDs, instead of picking the online vendor offering the lowest price, they tended to choose the branded vendors who charged a higher price. In addition to explanations associated with trust and convenience, choice overload might also be a major factor contributing to this behavior. Consumers' limited committed cognitive capacity prevented them from examining too many choices, and they relied on heuristics to simplify the task. In this case, they used the branded vendor as a surrogate to simplify the decision-making. As a result, the agent was underutilized in decision making, and consumers chose to pay the premium to avoid risk.

Thus, on one hand, some evidence indicated that electronic decision aids like comparison-shopping agents can help online shoppers by reducing search and comparison cost. On the other hand, the same decision aids may also overload online shoppers by providing too many choices. This paradoxical phenomenon of electronic decision aids could pose serious issues for the design of such decision aids - How to make online shoppers feel there are enough choices, while, at the same time, not overloading them? If too few, the owners of electronic decision aids may lose potential revenues from online sellers; if too many, the owners may lose potential sales opportunities through online shoppers. This research is designed to explore this paradoxical nature of electronic decision aids by constructing a research model and propose seven hypotheses for testing its validity.

\section{The Research Model and Hypothesis}

To explore paradoxical phenomenon of electronic decision aids, we propose a research model based on [10] with several revisions. These revisions are based on the new features of agent-mediated online shopping environment. These new features include the higher level of richness of product information like attributes information, the way comparison information is presented by the agent and processed by online shoppers, the availability of electronic decision aids, and the quick update of comparison information in certain online environments.

Our research model consists of two parts, as indicated in figure 1 . On the left side is the agent-mediated online shopping environment, where electronic decision aids mediate the display format of the shopping task and the shopping task is measured by three independent variables: the number of alternative choices, the number of attributes for each choice, and the refreshing rate of the choice task. On the right side is the online shopper, whose shopping behavior is mediated by his or her domain knowledge about the products. The central concept, choice overload, is detected and measured by three dependent variables, decision quality, decision time, and decision confidence as well as satisfaction.

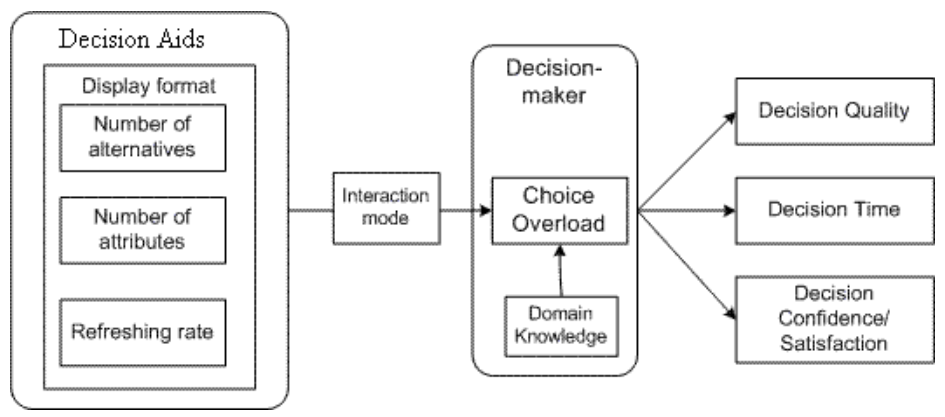

Figure 1: The Research Model

The richness and complexity of choice information in an agent-mediated environment was dramatically increased. In addition to providing many choice alternatives, specific attributes like price, physical dimension of the products, customer reviews, and ratings are also readily available for each alternative. They are explicitly presented and organized by electronic decision aids like comparison-shopping agents for comparison.

Such richness of information and the complexity to synthesize and balance them to make a selection may exceed the cognitive efforts that were needed in a traditional environment. The sheer amount of information may demand extra efforts from online shoppers to comprehend and make rational comparisons. This created a similar or even 
more severe environment that may incur choice overload among shoppers compared with those in [10]. And the amount of cognitive effort needed is directly related to the number of choices and number of attributes information provided in the decision task. When the overall size leads to choice overload status, online shoppers may exhibit symptoms like a decreasing quality of decision outcomes and less decision confidence and satisfactions. Thus, we have the following hypotheses:

H1: Oversupplying choice alternatives for online individual decision-making could increase the chance of choice overload and decision time, and decrease decision quality and confidence.

H2: Oversupplying attribute information for online individual decision-making could increase the chance of choice overload and decision time, and decrease decision quality and confidence.

As indicated in [10], shoppers' domain knowledge may implicitly influence choice overload. The more products domain knowledge an online shopper has, the more efficient and less effortful for him or her to comprehend and synthesize the information provided in the choice task about the product; thus, we have the following hypothesis about the mediating effect of domain knowledge on choice overload:

H3: The more familiar the decision-maker is with the product being investigated, the less the chance of choice overload when the number of attributes increases. Specifically, familiarity can increase decision quality and confidence, and decrease decision time.

In an agent-mediated environment, all information is preprocessed and presented in a predefined display format for comparison. And the display format of a choice set could influence the decision outcome [13], [31]. For example, Russo [19] found that by listing the unit price for each item on a shelf tag, decision efficiency and effectiveness could be greatly improved, and it could not only reduce the cost to the consumer but also generate more profit for the retailer. In an agent-mediated environment, on one side, online shoppers are enabled by electronic decision aids to manipulate the information display format to a certain extent [30]. On the other side, the display format will directly affect the priority, efficiency, and effectiveness of the information being processed.

The existence of electronic decision aids differentiates the online shopping environment from traditional environments as those in [10]. In a traditional environment, the shoppers had little assistance from the outside when they experienced choice overload. In an agent-mediated environment, there are decision tools like sorting and shortlisting available. The short-listing tool allows a shopper to reduce the number of choices for consideration. The sorting tool allows shoppers to ranking the choices based on specific attributes. A shopper could adjust the display of choice task by first using short-listing to reduce the number of choices and then using the sorting to rank the choice based on one's preference for certain attributes. Both of them may potentially reduce the decision efforts of shoppers. Thus, we have hypothesis 4:

H4: Providing sorting and short-listing features in agent-mediated online shopping environment increases decision quality, reduces decision time, and increases decision confidence and satisfaction.

Since sorting and short-listing are two complementary decision tools, we expect their combined use will give more benefit to shoppers than the use of only one. Thus, we have hypothesis 5 :

H5: In online individual decision-making, when two complementary decision tools (sorting and short-listing) are provided, one could achieve higher decision quality, use less decision time, and have higher decision confidence as well as decision satisfaction compared with the situations when only one of the decision tools is provided.

Shopping information like price and availability in an agent-aided online environment is usually updated in real time. The decision-makers may find the comparison information is no longer accurate if they refresh their choice task displayed in a Web page after a period of time has elapsed. This is especially true for some innovative B2C ecommerce models like Woot.com and countdown shopping offered by many online retailers, on which the availability of products could be updated in seconds. So it is interesting to know if it will increase the chance of choice overload when choice information is refreshed in real time combined with a large choice task. Knowing the choice information might be updated in a short while may pose anxiety to online shoppers, adding extra cognitive efforts to the same individual and potentially reducing the time commitment allocated for existing choices. These combined effects might lead to choice overload. Thus, we have hypothesis 6 :

H6: The increase of the choice information refreshing rate in online individual decision-making could increase the chance of choice overload by increasing decision time, decreasing decision quality, and confidence as well as satisfaction.

Finally, since the availability of decision tools such as sorting and short-listing might reduce the decision efforts and mitigate choice overload, we expect it will mitigate the negative effect posed by increasing the information refreshing rate. Thus, we have hypothesis 7 : 
H7: Sorting and short-listing can effectively reduce the chance of choice overload caused by the increase of the refreshing rate in online individual decision-making.

\section{The Methodology}

To validate our research model and hypotheses, we created a simulated comparison-shopping environment similar to the commercial comparison-shopping agents available on the Web.

\subsection{The Simulated Environment}

This simulated agent environment provided a comparison task for two product categories: digital cameras (DCs) and personal digital assistants (PDAs). For each product category, the simulated agent provides varying task sets. The complexity varies, ranging from " 4 choices under one brand with 5 product attributes for each choice" to "40 choices under 10 brands with 10 product attributes for each choice."

The simulated agent also provided sorting and short-listing (Figure 3) tools for subjects to use during their decisionmaking. The sorting decision tool was used to sort and rank all choices according to any available product attributes. It was executed by clicking the corresponding attributes on the first row of the decision task.

The short-listing decision tool was used to remove unwanted choices. Subjects could screen choices by selecting the check-box and then clicking the Compare button on top to remove unwanted (unchecked) choices.

There was also a background monitoring application that recorded subjects' activities, including the time spent at each stage of the decision-making process as well as the choices selected in each cycle. A post-experiment questionnaire was administered for each experiment to measure decision confidence and decision satisfaction as well as other subjective feelings.

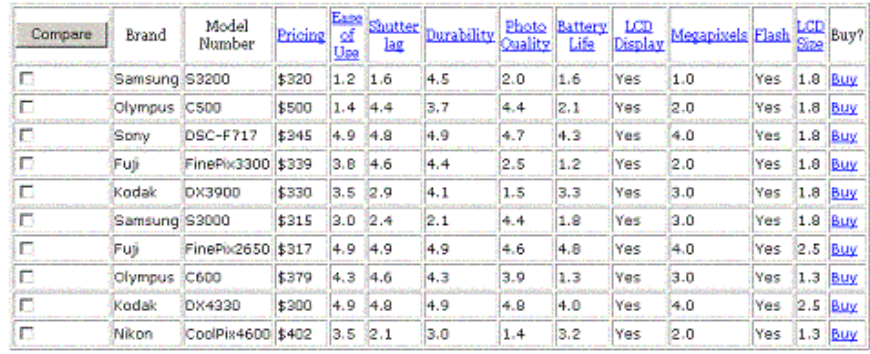

Figure 2: A Sample Decision Task Interface

\subsection{The Measurement of Dependent Variables}

To measure the operational variables in choice overload, we used decision quality, decision time, decision confidence, and decision satisfaction as dependent variables. The design of measurement for each variable is described below.

\subsubsection{Decision Quality}

Decision quality is an objective variable used to measure the impact of the comparison-shopping agent on choice overload. Since the quality of a choice could be subjective if a trade-off has to be made among them, each decision task was designed in such a way that it is objectively possible to classify it into either an optimal or non-optimal decision. The methodology adopted by Häubl and Trifts [9] was used to achieve this goal. Specifically, we designed two levels of decision quality for all choice candidates-the dominant level and the dominated level. Each choice alternative belonged to only one of the categories. For each brand of the product, there was only one dominant choice and several dominated choices. For the dominant choice, all its attributes are better than or at least as good as the other choices under the same brand. For the dominated choices, at least one of the attributes is not as good as the dominant choice under the same brand. So, for whichever brand, as long as the decision-maker selected the dominant choice for this brand, decision quality was optimal. If the decision-maker selected a dominated choice, the decision quality was not optimal.

\subsubsection{Decision Time}

Decision time is the amount of time the decision-maker takes to make a decision. In this study, we measured the amount of time a subject spent on each page and decision-task adjustment from the moment the subject entered the 
experiment site and began to read the decision task explanation until the final decision was made and the subject exited the site. We use $t_{\text {enter }}$ to indicate the moment the subject entered the decision task page and $t_{\text {exit }}$ to indicate the moment the subject left the site, so decision time is calculated as $t_{\text {decision }}=t_{\text {exit }}-t_{\text {enter }}$.

\subsubsection{Decision Confidence and Decision Satisfaction}

Decision confidence and decision satisfaction are defined as the degree of confidence and satisfaction a subject has toward the decision process and decision outcome. They were measured by questionnaires after the decision task was completed. At least three questions were used to measure the same construct and then Cronbach alpha was used to determine the reliability of each measurement.

\section{The Experiments and Results}

The purpose of the first experiment was to detect the range and pattern of task size that may trigger choice overload in two product categories: digital cameras (DCs) and personal digital assistants (PDAs).

\subsection{Experiment I}

Though these two products are both familiar to college students, we expected a difference in the level of familiarity between the two product categories. We found that digital cameras were comparatively more widely used than PDAs among college students. However, this needs to be verified in the experiment.

\subsubsection{Experiment Design}

The experiment used a between-subjects design of 4 (number of choices: 12, 20, 32, 40) x 2 (number of attributes: 5 , 10) $\times 2$ (digital camera and PDA) to present subjects with variously sized choice tasks. Each choice task consisted of $n$ number of brands $(n=3,5,8,10)$. For each brand, there were 4 similar product models with different attribute values. There was only one dominant product in each brand to allow us to identify decision quality with whatever brand a subject preferred.

We included short-listing as a decision tool in this stage of the experiment for two reasons. First, a comparisonshopping agent usually provides at least one decision tool to facilitate decision-making. Since in this experiment a decision task could be as big as $40 \times 10$, short-listing allowed the subject to conduct a prescreening so as to reduce the chance of choice overload to a certain level. Second, additional sorting tools at this stage could mitigate the effect of choice overload from task size. By providing only short-listing as a decision tool, the demand for cognitive effort in the decision task would be measured in between decision tasks that have no decision tools supporting them and those having the support of two decision tools.

\subsubsection{Subjects}

We recruited college students from a large freshman-level MIS class. To encourage subjects' participation in the experiment, each participant received two extra credits toward his or her final grade. In total, there were 224 subjects who participated in this experiment (104 subjects for digital cameras and 120 subjects for PDAs). Because some subjects quit the experiment prematurely, we finally got 97 complete data points for both digital cameras and PDAs.

\subsubsection{Procedure}

At the beginning of the experiment, subjects were introduced to the procedure of the experiment. They were asked to volunteer their university e-mail address for extra credit. Each subject was directed to a simulated comparisonshopping agent mediated environment. They were given a sample decision task page to familiarize themselves with the process, and then they were directed to the formal choice task page.

The choice task each subject received varied and was in one of the 16 conditions. Each subject was randomly assigned to one of these conditions. For each condition, there were 10-15 subjects. At any stage of the decisionmaking, including the beginning, subjects could select a subset of the choice set through the short-listing function for a more detailed comparison. When they identified their final choice, subjects clicked the Buy link for the choice and completed the task. The estimated time for each subject was 15 to 20 minutes.

After the decision was made, subjects were directed to a questionnaire page to get their subjective ratings on decision confidence, task difficulty, product familiarity, and other individual differences. We did not test decision satisfaction in this stage of the experiment. 


\subsubsection{Data Analysis}

We first used $F$ test to detect the between-subject effects. To analyze the trend and the degree of impact of each independent variable, we used linear regression analysis to get their beta value. The summary statistics are organized in Table 1 and 2. The Cronbach alpha on the decision confidence response from the questionnaires was 0.7632 , so we can average the responses to form a reliable scale to measure the construct of decision confidence.

From the statistics summarized in Table 1 and 2, we found that though increasing the number of choices will lead to decreasing decision quality and increasing decision time as indicated in Table 2, such trends were not statistically significant. Specifically, increasing the number of choices only has a marginally significant effect on the decreasing of decision quality $(p=0.076)$ and no effect on decision confidence. Thus, hypothesis 1 was not supported.

The between-subject effects data indicates number of attributes had a significant effect on the decision quality $(p=0.012)$. Regression analysis indicates when the number of attributes increases, decision quality decreases significantly $($ Beta $=-0.172, p=0.013)$. The effect on decision time and confidence are not significant. Thus hypothesis 2 was partially supported.

Domain knowledge has significant impact on decision quality, time, and confidence. 13 out of 97 subjects indicated that they had purchased a digital camera before, while only 3 out of 97 indicated they had purchased a PDA before. We used a cross-tab test and found that the proportion of ownership was significantly different between these two product categories, and owners of digital cameras were significantly higher in proportion to owners of PDAs (Pearson Chi-square Asymp Sig. $=0.009$ ). Subjects were more familiar with digital cameras than PDAs, and their domain knowledge for digital cameras was richer than for PDAs. And both between-subject effects and regression analysis indicated that more domain knowledge leads to increasing decision confidence, decreasing decision time, and increasing decision confidence. Thus, hypothesis 3 was fully supported.

We further analyzed the mediating effect of decision time to determine if it was reasonable to assume that the more time a subject spend on the choice task, the higher the quality of the final decision. To explore this effect, we included decision time as a covariate in the regression analysis model. The result indicated that decision time highly correlated with decision quality (Beta $=0.266, p=0.000$ ). This means the more time subjects spend on the choice task, the higher the decision quality. Together with the evidence of correlation between decision time and number of choices as well as number of attributes, we can predict that subjects spend more time on larger choice sets to retain the decision quality and compensate for the increasing complexity of the decision task. Through a $t$ test on the difference in mean between average decision time on both product categories, we found that the average decision time for PDAs (143.75 seconds) is significantly longer than for digital cameras (104.25 seconds) and the $p$-value for two-tail $t$ test on mean is 0.04 . This is in accordance with the self-reporting by the majority of the subjects that they are more familiar with digital camera than PDA.

Table 1: Between-Subjects Effects for Decision Quality, Time, and Confidence in Experim
\begin{tabular}{|c|c|c|c|c|c|c|}
\hline & Decision Quality & Decision Time & \multicolumn{2}{c|}{ Decision Confidence } \\
\cline { 2 - 8 } & F-Value & p-Value & F-Value & p-Value & F-Value & p-Value \\
\hline Number of Choices & 2.331 & 0.076 & 1.669 & 0.175 & 1.135 & 0.336 \\
\hline Number of Attributes & 6.407 & $0.012^{*}$ & 1.398 & 0.239 & 0.014 & 0.906 \\
\hline Domain Knowledge & 8.879 & $0.003^{*}$ & 4.470 & $0.036^{*}$ & 16.308 & $0.000^{*}$ \\
\hline
\end{tabular}


Table 2: Coefficients for Decision Quality, Time, and Confidence in Experiment I

\begin{tabular}{|c|c|c|c|c|c|c|c|c|c|}
\hline & \multicolumn{3}{|c|}{ Decision Quality } & \multicolumn{3}{c|}{ Decision Time } & \multicolumn{3}{c|}{ Decision Confidence } \\
\cline { 2 - 10 } & Beta & t-Value & p-Value & Beta & t-Value & p-Value & Beta & t-Value & p-Value \\
\hline Number of Choices & -0.134 & -1.958 & 0.052 & 0.118 & 1.670 & 0.097 & 0.046 & 0.665 & 0.507 \\
\hline Number of Attributes & -0.172 & -2.519 & $0.013^{*}$ & 0.088 & 1.232 & 0.219 & 0.009 & 0.130 & 0.896 \\
\hline Domain Knowledge & 0.207 & 2.996 & $0.003^{*}$ & -0.152 & -2.146 & $0.033^{*}$ & 0.281 & 4.023 & $0.000^{*}$ \\
\hline
\end{tabular}

We also analyzed the estimated marginal means of decision quality and time on the two product categories.

From the plotting of estimated marginal means for decision quality in Figure 3, we can see similar trends of declining marginal means in decision quality with the increasing number of choices and attributes. When the number of choices is over 20 , decision quality is almost constant. Thus, the choice overload threshold was probably beyond 20 for most subjects. The impact of increasing the number of attributes on decision quality was significant. When the number of attributes increased from 5 to 10, the marginal means of decision quality dropped significantly. This pattern is similar in both product categories.

From the plotting of estimated marginal means for decision time in Figure 4, we found that decision time increased in proportion to an increasing number of choices, and, then, there was a sudden drop in the 40 choices range. It is quite possible that when faced with so many choices, subjects simply give up on considering each choice, an indicator of choice overload. Also, with the increasing number of attributes, the marginal means of decision time also increased significantly for both product categories.

Overall, through experiment I, we found partial support for $\mathrm{H} 1$ to $\mathrm{H} 3$. In summary, with the increasing number of choices and attributes for each choice, the decision quality and decision time are either influenced significantly or marginally. Specifically, both decision quality and decision time are more susceptible to increasing the number of attributes for each choice compared with increasing the number of choices. The domain knowledge of subjects on the products has significant influence on all dependent variables, including decision quality, decision time, and decision confidence.

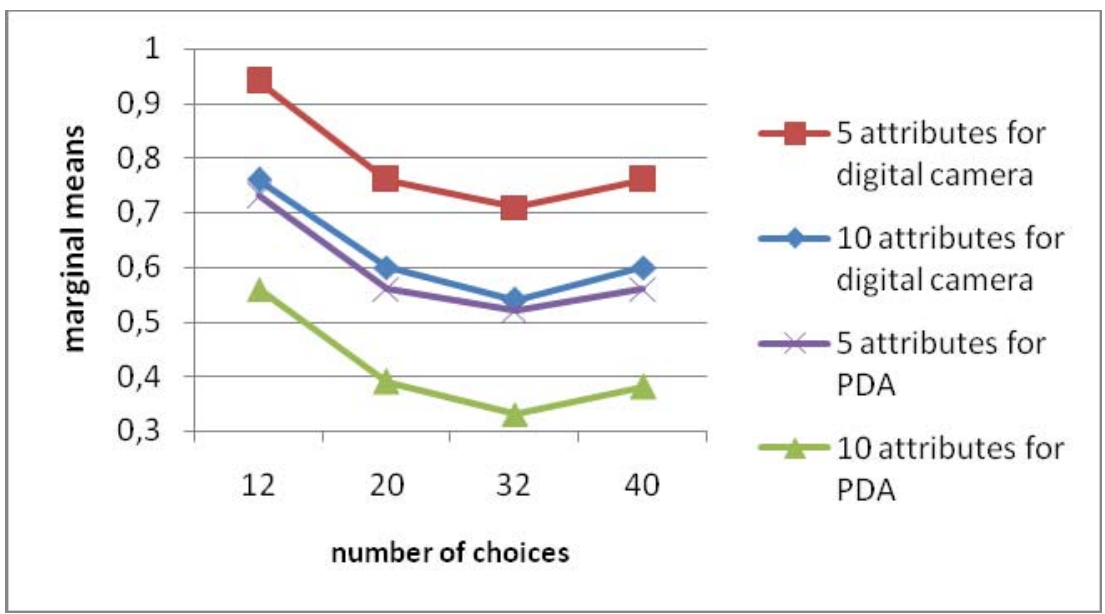

Figure 3: Estimated Marginal Means of Decision Quality in Experiment I 


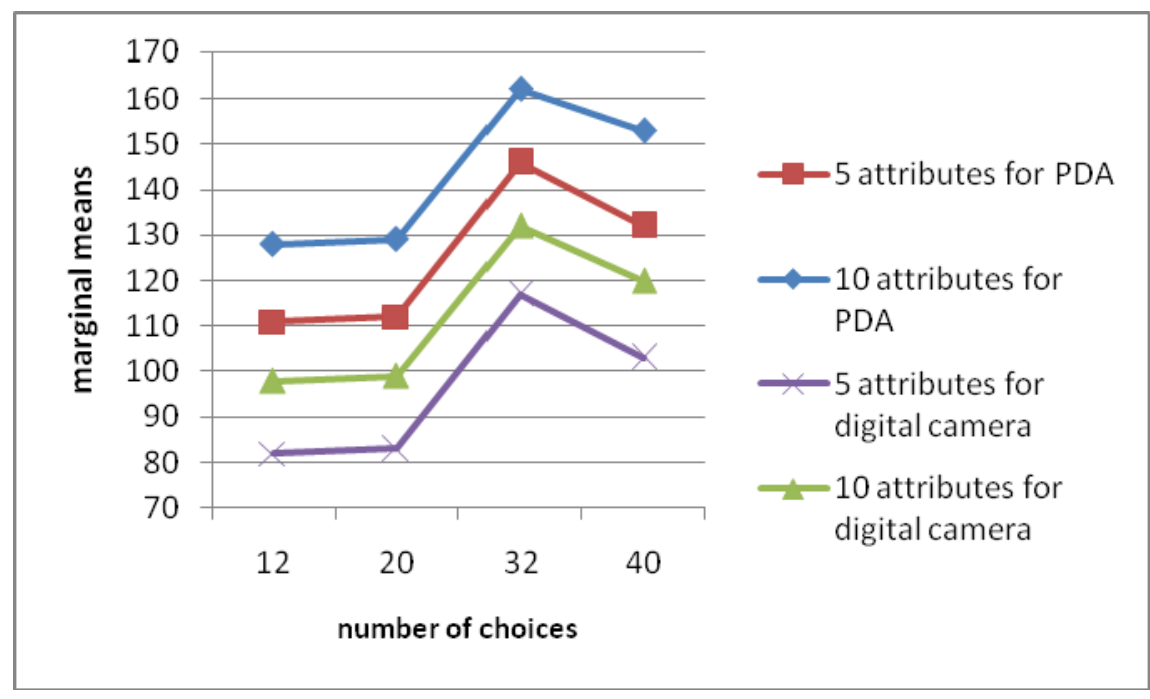

Figure 4: Estimated Marginal Means of Decision Time in Experiment I

By plotting the marginal means of decision quality and decision time for each decision task separately, we found there is a possible "choice overload" task size range when the number of choices exceeds 20 and the number of attributes exceeds 10 . Subjects reach a turning point in terms of decision quality and decision times beyond this combination of size. To explore whether decision tools embedded in electronic decision aids could mitigate the impact of this overload, we designed the second experiment.

\subsection{Experiment II}

With the estimation that the range of task size that exceeds $20 \times 10$ could trigger choice overload that we discovered in experiment $\mathrm{I}$, we created the second experiment.

\subsubsection{Experiment Design}

The second experiment is designed with a fixed size of $24 \times 10$ brands and attributes. We used digital cameras as the product for this experiment. We also added refreshing rate into this experiment. The experiment used a betweensubjects design of 2 (with vs. without short-listing tool) $\times 2$ (with vs. without sorting tool) $\times 2$ (with vs. without refreshing).

The short-listing tool allowed subjects to select their preferred products from the list by removing unwanted choices by checking the box on the left for the choices and then clicking Compare to filter out unchecked choices. The sorting tool provided the sorting/ranking features for all attributes of the products. The subject clicked the attribute of the products, and selection lists were redisplayed according to the ranking of attributes. The refreshing feature updated the existing comparison list every 60 seconds. In each update, the price for the product was changed according to predefined data.

\subsubsection{Subjects}

In the second experiment, we recruited 252 college students and received 240 complete sample data. The subjects were told to buy a digital camera that offered the best value for the price. To increase participation, subjects were told they had a chance of winning a $\$ 50$ cash award if they made decisions of good quality.

\subsubsection{Procedure}

Subjects were recruited via an e-mail announcement on a university mass mail and through several cooperative instructors. Subjects were provided a URL in their browser to go to the experiment page. In the first few pages, subjects were introduced to the procedures of the experiment. Depending on different scenarios, subjects were introduced to decision tools and/or refreshing features they would be able to use during the experiment, then they were directed to a simulated agent environment.

The specific decision task varied among eight conditions with different combinations of the two decision tools (shortlisting and sorting) as well as varying rates on the refreshing feature. After a decision was made, subjects were directed to a questionnaire page to get subjective ratings on confidence and satisfaction. Each construct was detected by 5 to 6 question items. The Cronbach Alphas for the two variables are 0.7503 and 0.8967 respectively. 


\subsubsection{Data Analysis}

After the data was collected, we conducted a data transformation: the total time spent on the experiment task was divided into one of two categories: review time and decision time. Review time was the time used to review the description of the decision task, which indicated the subject's preparedness in decision-making. Decision time was the actual time subjects spent making the selection, including using the decision tools to manipulate the choice set.

The data was analyzed by using hierarchical log linear analysis (K-way and higher order effects) and logistic regression. We found that though sorting could significantly increase decision quality $(B e t a=0.877, p$-value $=0.022)$, the effect of short-listing on decision quality was not significant. Using GLM full factorial analysis, we found that sorting could significantly decrease decision time while short-listing could significantly increase decision time. Specifically, the presence of a short-listing function increased decision time from an average of 141 seconds to 191 seconds, a $35 \%$ increase. There were no significant effects of both decision tools on decision confidence and satisfaction. Thus hypothesis 4 is partially supported.

When comparing data for the presence of both decision tools with only one of them or none of them present, there are no statistically significant differences. By comparing the means of each variable, we found that the presence of both decision tools actually led to worse decision quality than when only one of them was available. In addition, the mean decision time was also longer when both decision tools were available rather than when only one of them was available (Table 3). But subjects did have higher degrees of decision confidence and satisfaction when both decision tools were present than when one of them was there (Table 4 and 5). In summary, hypothesis 5 is not supported.

Table 3: Comparison of mean decision time

\begin{tabular}{|c|c|c|c|c|}
\hline \multirow{2}{*}{ Shortlisting } & \multirow{2}{*}{ Sorting } & \multirow{2}{*}{ Mean } & \multicolumn{2}{|c|}{$95 \%$ Confidence Interval } \\
\cline { 4 - 5 } & & & Lower Bound & Upper Bound \\
\hline No & No & 207 & 150 & 263 \\
\hline No & Yes & 125 & 68 & 182 \\
\hline Yes & No & 176 & 119 & 232 \\
\hline Yes & Yes & 243 & 186 & 299 \\
\hline
\end{tabular}

Table 4: Comparison of mean decision satisfaction

\begin{tabular}{|c|c|c|c|c|}
\hline \multirow{2}{*}{ Shortlisting } & \multirow{2}{*}{ Sorting } & \multirow{2}{*}{ Mean } & \multicolumn{2}{|c|}{$95 \%$ Confidence Interval } \\
\cline { 4 - 5 } & & & Lower Bound & Upper Bound \\
\hline No & No & 5.694 & 5.313 & 6.076 \\
\hline No & Yes & 5.389 & 5.008 & 5.770 \\
\hline Yes & No & 5.278 & 4.897 & 5.659 \\
\hline Yes & Yes & 5.467 & 5.086 & 5.848 \\
\hline
\end{tabular}

Table 5: Comparison of mean decision confidence

\begin{tabular}{|c|c|c|c|c|}
\hline \multirow{2}{*}{ Shortlisting } & \multirow{2}{*}{ Sorting } & \multirow{2}{*}{ Mean } & \multicolumn{2}{|c|}{ 95\% Confidence Interval } \\
\cline { 4 - 5 } & & & Lower Bound & Upper Bound \\
\hline No & No & 5.773 & 5.236 & 6.310 \\
\hline No & Yes & 4.847 & 4.310 & 5.384 \\
\hline Yes & No & 5.093 & 4.556 & 5.630 \\
\hline Yes & Yes & 5.267 & 4.730 & 5.804 \\
\hline
\end{tabular}


We use GLM full factorial analysis to further explore the effect of the information refreshing feature when coupled with decision tools. We found that increasing the refreshing rate significantly decreased decision quality. When the sorting feature was present, the decision quality increased by an odd-ratio of 2.4. The presence of the refreshing feature on the decision task decreased decision quality by an odd-ratio of 0.48 . The odd-ratio for the short-listing function was not significant though we were able to see it increase the chance of a better decision by an odd-ratio of 1.07 .

However, instead of increased decision time, for almost all group peers the presence of the refreshing feature decreased decision time from 187 seconds to 144 seconds on average compared with their counterparts who where without refreshing feature. It is possible that under the time pressure from information refreshing, subjects were intentionally speeding up the decision process (see Payne et al. [25]). The only exception was in groups with only sorting tools available. For the two groups with only sorting tools, the group without refreshing condition used less decision time than the group with the refreshing condition. This exception may come from the design of the experiment because in each brand group, there was only one dominant choice. The subject could very easily identify the dominant choice when using sorting, which actually ranked the choices. However, when the short-listing function was also available, subjects used it to do a more intensive decision analysis, which could be interrupted by information refreshing, thus increasing decision time, though it may not have necessarily led to better decision quality.

Table 6: Comparison of mean decision time when information is refreshing

\begin{tabular}{|c|c|c|c|c|}
\hline \multirow{2}{*}{ Shortlisting } & \multirow{2}{*}{ Sorting } & \multirow{2}{*}{ Mean } & \multicolumn{2}{|c|}{$95 \%$ Confidence Interval } \\
\cline { 4 - 5 } & & & Lower Bound & Upper Bound \\
\hline No & No & 87 & 30 & 143 \\
\hline No & Yes & 146 & 89 & 202 \\
\hline Yes & No & 152 & 95 & 209 \\
\hline Yes & Yes & 193 & 136 & 250 \\
\hline
\end{tabular}

In terms of decision confidence and satisfaction, increasing the refreshing rate decreased decision confidence and decision satisfaction while the availability of decision tools could mitigate such effects.

Table 7: Comparison of mean decision satisfaction when information is refreshing

\begin{tabular}{|c|c|c|c|c|}
\hline \multirow{2}{*}{ Shortlisting } & \multirow{2}{*}{ Sorting } & \multirow{2}{*}{ Mean } & \multicolumn{2}{|c|}{$95 \%$ Confidence Interval } \\
\cline { 4 - 5 } & & & Lower Bound & Upper Bound \\
\hline No & No & 5.140 & 4.603 & 5.677 \\
\hline No & Yes & 5.427 & 4.890 & 5.964 \\
\hline Yes & No & 5.227 & 4.690 & 5.764 \\
\hline Yes & Yes & 4.713 & 4.176 & 5.250 \\
\hline
\end{tabular}

Table 8: Comparison of mean decision confidence when information is refreshing

\begin{tabular}{|c|c|c|c|c|}
\hline \multirow{2}{*}{ Shortlisting } & \multirow{2}{*}{ Sorting } & \multirow{2}{*}{ Mean } & \multicolumn{2}{|c|}{$95 \%$ Confidence Interval } \\
\cline { 4 - 5 } & & & Lower Bound & Upper Bound \\
\hline No & No & 5.183 & 4.802 & 5.564 \\
\hline No & Yes & 5.461 & 5.080 & 5.842 \\
\hline Yes & No & 5.206 & 4.824 & 5.587 \\
\hline Yes & Yes & 5.239 & 4.858 & 5.620 \\
\hline
\end{tabular}


We used GLM full factorial analysis and found that the presence of only one decision tools generally increased decision satisfaction and confidence. However, such differences are not significant. The only significant interaction effect was that subjects have the lowest decision satisfaction when both decision tools are available $(p$-value = 0.014). In other words, though the presence of one of the decision tools increased the decision satisfaction, when both decision tools were available, the decision satisfaction decreased.

In summary, increasing the information refreshing rate will decrease decision quality, decrease decision time in most cases, and decrease confidence as well as satisfaction. Thus Hypothesis 6 is partially supported. Also, the presences of sorting and short-listing could increase decision quality but cannot decrease decision time. There is also no significant effect on increasing decision satisfaction and confidence, though there is a trend of that. Thus, Hypothesis 7 is partially supported.

As indicated in previous analysis, when both decision tools were present in the information refreshing condition (we called it "hypermedia" context), subjects had the lowest decision satisfaction. In a further exploration of data from experiment II, we found that, on the other extreme, when no decision tool was available and there was no information refreshing (we call it "traditional media" context), subjects had the highest satisfaction. Considering the general scenario these two contexts represent (the former is typical interactive Web environment while the latter is typical traditional paper media), it is important to understand why subjects had this counter-intuitive reaction.

We examined the comments they wrote after answering the questionnaire. We found two interesting differences in the comments. First, far fewer subjects chose to make comments in the hypermedia group compared to the traditional media group. In addition, almost $50 \%$ of subjects who left comments in the hypermedia group mentioned their anticipation of product pictures in comparison-shopping compared with less than $15 \%$ in the traditional media condition.

This difference indicated that subjects in the hypermedia context probably treated the choice task as an online decision task, thus having high expectation on the information richness, and they were seeking additional comparison information to make their decisions even though they were not available. In the traditional media condition, because of a lack of any decision tools to allow subject sorting and short-listing, subjects probably treated the choice task more like reviewing a page of a catalog magazine. Also they were probably overwhelmed by the comparison information already provided and felt less willing to acquire additional comparison information such as product pictures.

\section{Discussion}

One goal of this research was to explore the paradoxical nature of electronic decision aids in assisting online decision-making: they are supposed to reduce the cognitive effort of decision-makers; however, the actual effect might be the opposite.

\subsection{The Detrimental Effect of Electronic Decision Aids: Choice Overload}

In the first experiment, we demonstrated that oversupplying information increased the likelihood of choice overload, which confirmed hypotheses 1 and 3. Specifically, we found that decision quality decreased abruptly when the number of choices increased from 12 to 24 or more. In addition, decision quality decreased significantly when the number of attributes increased from five attributes to 10 in all contexts. We found that increasing the number of attributes had a comparatively higher impact on choice overload than increasing the number of choices. Agent designers should pay attention to the introduction of new product and service features for comparison. In addition, a concise and clear explanation for each product attribute is always desirable for online shoppers not familiar with the product.

Choice overload was observed in the increased decision time when the number of choices and attributes increased. However, there was a sudden drop in the amount of decision time being spent on decision-making when the number of choices increased from 36 to 48 in both product categories. Usually, when facing too many choices, subjects defer their decision or choose the default option because of choice overload [22]. However, in our experiment, there was no option to defer decision-making, so subjects arbitrarily chose an alternative and finalized their decision in 48choice contexts.

Domain knowledge also played a role in choice overload. For the two products we used for experiment I, digital cameras were more familiar to subjects than PDAs. Overall, decision quality for digital cameras was significantly higher than for PDAs. The decision time for digital cameras was significantly higher than for PDAs, too, so subjects spent less time considering PDAs. Relating this finding to the lower familiarity with PDAs, it seems when consumers are faced with unfamiliar products, they tend to use less decision time and engage heuristics, which could lead to low decision quality and less confidence. Domain knowledge was the only marginally significant variable in decision confidence. When we reduced all insignificant interaction variables, domain knowledge was the only significant variable $(p=0.001)$, so we concluded that the confidence of decision-makers in a choice overload context was 
mainly determined by their familiarity with the product or their domain knowledge. This finding is complementary to findings in [10], though they focused only on decision satisfaction.

Experiment I indicates an important pattern in decision behavior that could indicate choice overload and possibly extend to other contexts; this was the abrupt decrease in decision time. In an online environment, the major cognitive demand is processing information. When the choice task size increases, a reasonable amount of time is spent on processing if there is no choice overload. When the decision time decreased abruptly, choice overload was almost certainly the cause and that heuristics is used instead. Heuristics reduces decision time and effort but it also reduce decision quality as the consumer makes a random selection. Other online shopper behaviors such as shopping cart abandonment could be developed from this knowledge.

\subsection{The complexity of impact of decision tools on mitigating choice overload}

The purpose of the second experiment was to explore the efficiency and effectiveness of decision tools on mitigating choice overload. Several findings from this experiment warrant further investigation. First, the sorting function has significant influence on decision quality compared to the short-listing function. Sorting increased decision quality, in part because of the structural design of the data source, i.e., there was one dominant choice in each brand and this choice had dominant value in each attribute, so it was relatively easy for subjects to make a dominant choice. However, when this strategy is used, decision time should not change significantly with an increased number of choices.

Second, short-listing tools increased decision time, contrary to expectations. The purpose of a short-listing function is to enable users to quickly identify the choices they prefer and narrow the field so as to make the decision more efficiently. However, our results generally indicated the opposite, possibly because short-listing induces noncompensatory strategies and compromises the decision confidence of the consumer. The confidence deficiency increased with the size of the decision task. At a critical stage, consumers may have doubted the results from the short-listing function and redid it, increasing their decision time.

Third, the availability of more decision tools may not lead to higher decision quality. Instead, it may lead to less decision satisfaction.

Because the use of decision tools by different users varies significantly, when more than one decision tool was provided, decision quality did not necessarily increase. According to the least effort principle, decision-makers may only seek a so-called "satisficing $[23,24]$ " decision outcome. When the use of multiple decision tools required more effort, decision-makers may not use them in an effective way at all, leading to even less decision satisfaction and a lack of confidence to the point of seeking additional comparison information that was not provided. Future research should focus on the complementary effect of multiple decision functions in reducing choice overload and the psychological consequences of decision satisfaction and confidence for the consumer.

\subsection{The Influence of Time Pressure}

The second experiments made it clear that refreshing conditions reduce decision time and quality. Initially, we assumed decision time would increase in these circumstances. However, it turned out that when subjects were aware that the information was updated during their decision process, they actually increased their decision-making rate to avoid reconsidering the updated information, similar to what people do in the stock market. Decreased decision time led to decreased decision quality, as expected.

In summary, this research identified the existence of choice overload in agent-mediated online individual decisionmaking. We also found the domain knowledge of the decision-maker is critical for mitigating choice overload in an agent-mediated environment. Another important finding is that even when an agent provides decision tools for a decision-maker, the aggregated effect is not necessarily improved efficiency and effectiveness of the decision process. The impact of decision tools and functions provided by electronic decision aids on decision outcomes needs further exploration.

\section{Conclusions, Limitations, and Future Research}

In this research, we explored how electronic decision aids influence online individual decision-making by providing varying sizes of decision tasks and decision-supporting functions. There are two major contributions of this research: a relatively comprehensive theoretical analysis on the choice overload phenomenon in comparison-shopping agentmediated online decision-making, which can be a reference for future research in this area, and the impact of the two most widely used decision-supporting tools (short-listing and sorting) on mitigating choice overload. Decision tools such as these two that mainly support non-compensatory decision strategies do not necessarily improve the efficiency and effectiveness of decision-making as expected. Specifically, when both of them were available, the 
combined effect in reducing decision time and effort and in improving decision satisfaction was not necessarily better compared with the situation when only one was available.

\subsection{Empirical Applications}

There are important empirical applications of this study.

For comparison-shopping service providers, this study indicates merely listing more choices and additional attribute information may not necessarily result in more click-through. Adding more sophisticated decision tools is also not necessarily helping online shoppers overcome choice overload. It is the combination of appropriate choice set size and orchestration of various decision tools that works.

For online vendors, this study indicates using the most popular comparison-shopping services may not necessarily lead to better sales outcome. More sophisticated pricing and service combination strategies are needed for online vendors to compete for the limited attention of online shoppers [32], [35].

\subsection{Limitations}

There are several major limitations of this research. First, since we are still lacking a satisfactory explanation of heuristic behavior, the internal validity of this research was restricted by the validity of its underlying assumptions, e.g., the bounded rationality of decision-makers and the adaptation of the brain as described in evolution psychology. The external validity of this research was also limited due to the fact that we had only two product categories in our experiments. In real life, the behavior of online shoppers varies when they are making shopping decisions in different product categories. For example, online shoppers spend more time using a comparison-shopping agent to buy a car than they do to buy a digital camera or a PDA, even though they are processing the same amount of information. Since online shoppers would commit more effort to buying a car compared with buying a digital camera, the same choice set size that may overload a buyer for a digital camera may be just right for a car buyer, though for the latter, larger size may just as well lead to a similar overload result.

The experiment was conducted in a simulated environment and only alphanumeric comparison information was used. Many subjects commented after the experiment that the product image played an important role in their decisionmaking. Since including a product image would make it very difficult for us to separate the visual effect from other comparison information, we did not include an image of the products in the experiment. However, we admit that by providing a product image, quite different heuristics may be employed by consumers, and outcomes may be quite different. These subtle effects cannot be successfully simulated in this experiment. More research should be done to explore how product image affects choice overload.

\subsection{Future Research}

Two major research directions grow out of this research.

First, more sophisticated theoretical explorations of choice overload, preferably originating from decision heuristics and other descriptive models, are needed to design significantly better electronic decision aids equipped with appropriate decision tools. Recent studies have focused on identifying the existence of choice overload instead of how to use properly designed decision aids to prevent choice overload [10], [20] - [21]. Without properly understanding the interaction pattern between these decision aids and decision-makers, we can hardly substantiate their claimed decision efficiency and effectiveness to online shoppers. As mentioned in previous sections, choice overload was directly related to the commitment of effort by decision-makers. Future experiments could focus on two aspects: how much decision effort has been committed before the decision task and how much actual effort has been invested in the decision task. Then, by varying the size and category of product-comparison tasks, we could more accurately describe the happening of choice overload.

Second, further investigation of the impact of decision-supporting tools provided by decision aids on managing choice task is also needed. Future experiments could focus on the usage pattern for different tools with varying large choice tasks. There are a few studies on the impact of controls on decision-making ([2], [8] - [9]), but few explore the use of decision support tools in larger choice contexts. This research provides a starting point for these explorations. 


\section{References}

[1] J. Alba, J. Lynch, B. Weitz, C. Janiszewski, R. Lutz, A. G. Sawyer, and S. Wood, Interactive home shopping: Consumer, retailer, and manufacturer incentives to participate in electronic marketplaces, Journal of Marketing, vol. 61, no. 3, pp. 38-53, 1997.

[2] D. Ariely, Controlling the information flow: effects on consumers' decision making and preference, Journal of Consumer Research, vol. 27, no. 2, pp. 233-248, 2000.

[3] H. Beales, M. B. Mazis, S. C. Salop, and R. Staelin, Consumer search and public policy, Journal of Consumer Research, vol. 14, no. 1, pp. 11-22, 1981.

[4] E. Brynjolfsson, and M. Smith, Frictionless Commerce? A Comparison of Internet and Conventional Retailers, Management Science, vol. 46, no. 4, 2000.

[5] D. Clark, Shopbots Become Agents for Business Change, IEEE Computer, vol. 33, no. 2, pp. 18-21, 2000.

[6] D. Clark, Shopbots: Help or Hindrance?, IEEE Intelligent Systems, vol. 15, no. 2, pp. 8-9, 2000.

[7] R. B. Doorenbos, O. Etzioni, and D. S. Weld, A Scalable Comparison-Shopping Agent for the World Wide Web, in First International Conference on Autonomous Agents, Marina del Rey, California, 1997, pp. 39-48.

[8] G. Häubl, and K. B. Murray, Preference Construction and Persistence in Digital Marketplaces: The Role of Electronic Recommendation Agents, Journal of Consumer Psychology, vol. 13, no. 1\&2, pp. 75-91, 2003.

[9] G. Häubl, and V. Trifts, Consumer Decision Making in Online Shopping Environments: The Effects of Interactive Decision Aids, Marketing Science, vol. 19, no. 1, pp. 4-21, 2000.

[10] S. S. lyengar, and M. R. Lepper, When Choice is Demotivating: Can One Desire Too Much of a Good Thing?, Journal of Personality and Social Psychology, vol. 79, no. 6, pp. 12, 2000.

[11] E. J. Johnson, W. W. Moe, P. S. Fader, S. Bellman, and G. L. Lohse, On the depth and dynamics of online search behavior, Management Science, vol. 50, no. 3, pp. 299-308, 2004.

[12] D. Kahneman, P. Slovic, and A. Tversky., Judgment under uncertainty: heuristics and biases, New York, NY: Cambridge University Press, 1982.

[13] D. Krantz, Rational Distance Functions for Multidimensional Scaling, Journal of Mathematical Psychology, vol. 4, no. 226-245, 1967.

[14] P. Maes, Agents that reduce work and information overload, Communications of the ACM, vol. 37, no. 7, pp. 3040, 1994.

[15] L. A. Montgomery, K. Hosanagar, R. Krishnan, and K. B. Clay, Designing a Better Shopbot, Management Science, vol. 50, no. 2, pp. 189-206, 2003.

[16] E. L. Olson, and R. E. Widing II, Are Interactive Decision Aids Better Than Passive Decision Aids? A Comparison With Implications For Information Providers On the Internet, Journal of Interactive Marketing, vol. 16, no. 2, pp. 22-33, 2002.

[17] J. W. Payne, J. R. Bettman, and E. J. Johnson, The adaptive decision maker: New York, NY: Cambridge University Press, 1993.

[18] R. E. Pereira, Influence of query-Based Decision Aids on consumer Decision Making in Electronic Commerce, Information Resources Management Journal, vol. 14, no. 1, pp. 31-48, 2001.

[19] J. E. Russo, The value of unit price information, Journal of Marketing Research, vol. 14, pp. 193-201, 1977.

[20] B. Schwartz, The Paradox of Choice. New York: HarperCollins, 2004.

[21] B. Schwartz, A. Ward, J. Monterosso, S. Lyubomirsky, K. White, and D. R. Lehman, Maximizing Versus Satisficing: Happiness Is a Matter of Choice, Journal of Personality and Social Psychology, vol. 83, no. 5, pp. 20, 2002.

[22] H. A. Simon, A behavioral model of rational choice, Quarterly Journal of Economics, vol. 69, no. 1, p. $20,1955$.

[23] H. A. Simon, Model of Bounded Rationality, vol 3 Empirically Grounded Economic Reason, Cambridge, MA: The MIT Press, 1997.

[24] H. A. Simon, Rational choice and the structure of the environment, Psychological Review, vol. 63, pp. 129-138, 1956.

[25] I. Simonson, J. Huber, and J. W. Payne, The Relationship between Prior Brand Knowledge and Information Acquisition order, Journal of Consumer Research, vol. 14, no. 4, pp. 566-78, 1988.

[26] M. D. Smith, and E. Brynjolfsson, Consumer Decision-Making at an Internet Shopbot, Journal of Industrial Economics, vol. 49, no. 4, pp. 541-558, 2001.

[27] P. Todd, and I. Benbasat, Evaluating the Impact of DSS, Cognitive Effort, and Incentives on Strategy Selection, Information Systems Research, vol. 10, no. 4, pp. 356-374, 1999.

[28] P. Todd, and I. Benbasat, The Use of Information in Decision Making: An Experimental Investigation of the Impact of Computer-Based Decision Aids, MIS Quarterly, vol. 16, no. 3, pp. 373-393, 1992.

[29] P. A. Todd, An Experimental Investigation of the Impact of Computer Based Decision Aids on the Process of Preferential Choice, in Department of Commerce Vancouver: The University of British Columbia, 1988.

[30] A. Tversky, and D. Kahneman, Judgment under Undertainty: Heuristics and Biases, Science, vol. 185, no. 4157, pp. 1124-1131, 1974.

[31] A. Tversky, and J. E. Russo, Substitutability and similarity in binary choice, Journal of Mathematical Psychology, vol. 6, pp. 1-12, 1969. 
[32] Y. Wan, Comparison-Shopping as an Emerging Channel to Increase Web Visibility for Small- and Medium-sized Enterprises (SME) in the United States, in Global Electronic Business Research: Opportunities and Directions (N. A. Al-Qirim, Ed.). Hershey, PA: Idea Group, 2005, pp. 214-237.

[33] P. M. West, D. Ariely, S. Bellman, E. T. Bradlow, J. Huber, E. J. Johnson, B. Kahn, J. Little, and D. A. Schkade, Agents to the Rescue?, Marketing Letters, vol. 10, no. 3, pp. 285-300, 1999.

[34] R. E. Widing, and W. W. Talarzyk, Electronic Information Systems for Consumers: An Evaluation of ComputerAssisted Formats in Multiple Decision Environments, Journal of Marketing Research, vol. 30, no. 2, pp. 125-141, 1993.

[35] Y. Xu, and H.-W. Kim, Order Effect and Vendor Inspection in Online Comparison Shopping, Journal of Retailing, vol. 84, no. 4, pp. 477-486, 2008.

[36] G. K. Zipf, Human Behavior and the Principle of Least Effort: An introduciton to Human Ecology. Cambridge, MA: Addison-Wesley Press, 1949. 